\title{
The Clausius-Mossotti Phase Transition in Polar Liquids
}

\author{
S. Sivasubramanian, A. Widom, and Y.N. Srivastava ${ }^{\dagger}$ \\ Physics Department, Northeastern University, Boston MA USA \\ ${ }^{\dagger}$ Physics Department $\&$ INFN, University of Perugia, Perugia Italy
}

\begin{abstract}
The conventional Clausius-Mossotti polarization equation of state is known to be unstable for polar liquids having molecules with high polarizability. Room temperature water is an important example. The instability in the polarization equation of state is of the typical loop form requiring an "equal area" construction for studying the stable ordered phase. The ordered phase of a ClausiusMossotti polar liquid then consists of domains each having a net polarization. The polarization may vary in direction from domain to domain. The ordered phases are quite similar to those previously discussed on the basis of Dicke superradiance.
\end{abstract}

PACS numbers: 05.20.Jj; 64.60.-i; 64.70.Ja

\section{INTRODUCTION}

The Clausius-Mossotti 1, 2] polarization equation of state is quite often discussed in text books on electromagnetic theory [3, [4, [5] . The discussion is thought to give students a good physical idea about how microscopic models of dipole moments yield non-trivial macroscopic material dielectric constants $\varepsilon$. It is only sometimes mentioned that the Clausius-Mossotti model exhibits an instability hinting at a phase transition into an ordered polarized state. The unstable precursor to the phase transition is in fact self evident. The instability is particularly strong in polar liquids such as water. In fact, the Clausius-Mossotti model in its classic text book form indicates that water at room temperature and pressure is in an ordered polarization state.

The ordered state of polar liquids is described in the literature as exhibiting superradiance 6 , 7, 8, 9]. In the superradiant state, the polarization $\mathbf{P}$ has a mean value roughly constant in magnitude within an ordered domain. However, $\mathbf{P}$ may vary in direction as one goes from one ordered fluid domain to another ordered fluid domain. The theoretical derivation of superradiant domains normally starts from condensed matter quantum electrodynamics. Yet many insights into the nature of superradiant thermal equilibrium can be obtained from a classical electrodynamic viewpoint. Our purpose is to derive the polar liquid phase transition implicit in the Clausius-Mossotti model. Our arguments employ (apart from classical electrodynamics) the elementary statistical thermodynamics previously worked out by Debye and Langevin [10, 11].

In Sec. II, we briefly discuss the derivation of the Clausius-Mossotti local electric field (in Gaussian units)

$$
\mathbf{F}=\mathbf{E}+(4 \pi / 3) \mathbf{P} .
$$

In Eq.(1), $\mathbf{E}$ is the total electric field and $\mathbf{P}$ is the dipole moment per unit volume. In terms of the molecular polarizability $\alpha$ of a single molecule and the liquid volume $v$ per molecule, we shall derive the mean field ClausiusMossotti dielectric constant $\varepsilon$. In Sec. III, we discuss the region of thermodynamic stability of the Clausius-
Mossotti model. The known instability is often thought to be an embarrassment for the mean field theory. However, we argue that the Clausius-Mossotti instability is a signature of a physical phase transition into an ordered phase. The ordered phase has been previously discovered from the viewpoint of superradiance. The thermodynamic theories of both the ordered and disordered phases are discussed from the Clausius-Mossotti viewpoint in Sec. IV. The nature of a more complete quantum theory is explored in the concluding Sec. V.

\section{CLAUSIUS-MOSSOTTI LOCAL FIELDS}

Consider the Coulomb energy contained in a charge density $\rho$, i.e.

$$
U=\frac{1}{2} \iint \frac{\rho(\mathbf{r}) \rho\left(\mathbf{r}^{\prime}\right)}{\left|\mathbf{r}-\mathbf{r}^{\prime}\right|} d^{3} \mathbf{r} d^{3} \mathbf{r}^{\prime} .
$$

If the charge density is derived from a macroscopic polarization

$$
\rho(\mathbf{r})=-\operatorname{div} \mathbf{P}(\mathbf{r}),
$$

then integrating Eq.(2) twice by parts yields

$$
U=\frac{1}{2} \iint \mathbf{P}(\mathbf{r}) \cdot \mathrm{G}\left(\mathbf{r}-\mathbf{r}^{\prime}\right) \cdot \mathbf{P}\left(\mathbf{r}^{\prime}\right) d^{3} \mathbf{r} d^{3} \mathbf{r}^{\prime}
$$

The dyadic

$$
\mathrm{G}\left(\mathbf{r}-\mathbf{r}^{\prime}\right)=\operatorname{grad} \operatorname{grad}^{\prime} \frac{1}{\left|\mathbf{r}-\mathbf{r}^{\prime}\right|}
$$

obeys the trace condition

$$
\operatorname{Tr} \mathrm{G}\left(\mathbf{r}-\mathbf{r}^{\prime}\right)=-\nabla^{2} \frac{1}{\left|\mathbf{r}-\mathbf{r}^{\prime}\right|}=4 \pi \delta\left(\mathbf{r}-\mathbf{r}^{\prime}\right) .
$$

Eqs.(15) and (6) imply that

$$
\begin{aligned}
\mathrm{G}(\mathbf{R}) & =-\Delta(\mathbf{R})+\frac{4 \pi}{3} 1 \delta(\mathbf{R}), \\
\Delta(\mathbf{R}) & =\frac{3 \mathbf{R} \mathbf{R}-R^{2} 1}{R^{5}},
\end{aligned}
$$


where the $(4 \pi 1 / 3) \delta(\mathbf{R})$ term in Eq. (17) is present to enforce the trace condition in Eq.(6). From Eqs.(4) and (7), it follows that the Coulomb energy

$$
U=U_{\text {dipole }}+U_{\text {local }}
$$

where

$$
U_{\text {dipole }}=-\frac{1}{2} \iint \mathbf{P}(\mathbf{r}) \cdot \Delta\left(\mathbf{r}-\mathbf{r}^{\prime}\right) \cdot \mathbf{P}\left(\mathbf{r}^{\prime}\right) d^{3} \mathbf{r} d^{3} \mathbf{r}^{\prime}
$$

and

$$
U_{\text {local }}=\frac{2 \pi}{3} \int|\mathbf{P}(\mathbf{r})|^{2} d^{3} \mathbf{r} .
$$

Consider the work done by bringing in from infinity a small change in the charge density $\delta \rho=-\operatorname{div} \delta \mathbf{P}$. The change in Coulomb energy will be given in terms of the potential $\Phi(\mathbf{r})$ according to

$$
\begin{aligned}
\delta U & =\iint \frac{\rho\left(\mathbf{r}^{\prime}\right) \delta \rho(\mathbf{r})}{\left|\mathbf{r}-\mathbf{r}^{\prime}\right|} d^{3} \mathbf{r}^{\prime} d^{3} \mathbf{r} \\
& =\int \Phi(\mathbf{r}) \delta \rho(\mathbf{r}) d^{3} \mathbf{r} \\
& =-\int \Phi(\mathbf{r}) \operatorname{div} \delta \mathbf{P}(\mathbf{r}) d^{3} \mathbf{r} \\
& =\int \operatorname{grad} \Phi(\mathbf{r}) \cdot \delta \mathbf{P}(\mathbf{r}) d^{3} \mathbf{r} .
\end{aligned}
$$

The electric field $\mathbf{E}=-\operatorname{grad} \Phi$ may then be computed from the functional variation

$$
\begin{aligned}
\delta U & =-\int \mathbf{E}(\mathbf{r}) \cdot \delta \mathbf{P}(\mathbf{r}) d^{3} \mathbf{r}, \\
\mathbf{E}(\mathbf{r}) & =\mathbf{F}(\mathbf{r})-\frac{4 \pi}{3} \mathbf{P}(\mathbf{r}) .
\end{aligned}
$$

The local electric field $\mathbf{F}(\mathbf{r})$ is due directly to the dipoledipole interaction function $\Delta(\mathbf{R})$ in Eq.(7) via the variation

$$
\begin{aligned}
\delta U_{\text {dipole }} & =-\int \mathbf{F}(\mathbf{r}) \cdot \delta \mathbf{P}(\mathbf{r}) d^{3} \mathbf{r}, \\
\mathbf{F}(\mathbf{r}) & =\int \Delta\left(\mathbf{r}-\mathbf{r}^{\prime}\right) \cdot \mathbf{P}\left(\mathbf{r}^{\prime}\right) d^{3} \mathbf{r}^{\prime}
\end{aligned}
$$

The remaining $(4 \pi / 3) \mathbf{P}(\mathbf{r})$ term on the right hand side of Eq.(12) is due to the local polarization energy in Eq.(10), i.e.

$$
\begin{aligned}
\delta U_{\text {local }} & =\delta U-\delta U_{\text {dipole }} \\
& =-\int\{\mathbf{E}(\mathbf{r})-\mathbf{F}(\mathbf{r})\} \cdot \delta \mathbf{P}(\mathbf{r}) d^{3} \mathbf{r} \\
& =\frac{4 \pi}{3} \int \mathbf{P}(\mathbf{r}) \cdot \delta \mathbf{P}(\mathbf{r}) d^{3} \mathbf{r} .
\end{aligned}
$$

Eq.(14) implies the Clausius-Mossotti local field Eq.(11).

Now suppose that the individual fluid molecules exhibit a mean electric dipole moment response to a local field as described by the polarizability $\alpha$ via

$$
\overline{\mathbf{p}}=\alpha \mathbf{F} .
$$

The dipole moment per unit volume (corresponding to a volume $v$ per molecule of fluid) then reads

$$
\mathbf{P}=\frac{\overline{\mathbf{p}}}{v}=\frac{\alpha \mathbf{F}}{v}=\frac{\alpha}{v}\left(\mathbf{E}+\frac{4 \pi}{3} \mathbf{P}\right) .
$$

Thus

$$
\mathbf{P}=\chi \mathbf{E},
$$

with a polarization susceptibility

$$
\chi=\left(\frac{\alpha}{v-(4 \pi / 3) \alpha}\right) .
$$

In terms of the dielectric constant $\varepsilon$,

$$
\mathbf{D}=\mathbf{E}+4 \pi \mathbf{P}=\varepsilon \mathbf{E},
$$

one finds the Clausius-Mossotti prediction

$$
\varepsilon=1+4 \pi \chi=\left(\frac{v+(8 \pi / 3) \alpha}{v-(4 \pi / 3) \alpha}\right) .
$$

All that is needed to calculate $\varepsilon$ (in this model) is the polarizability $\alpha$ for an isolated molecule and the volume per molecule $v$ in the fluid.

\section{THERMODYNAMIC STABILITY}

Landau and Lifshitz 12 prove a non-trivial theorem in their treatise on the electrodynamics of continuous media. The theorem asserts that the dielectric constant of a material obeys the inequality

$$
1<\varepsilon<\infty \quad \text { (thermodynamic stability). }
$$

We note (in passing) that the magnetic analogue of the theorem (with $\mathbf{B}=\tilde{\mu} \mathbf{H}$ ) asserts only the weaker magnetic permeability inequality $0<\tilde{\mu}<\infty$. Thus, diamagnetism $(0<\tilde{\mu}<1)$ can exist but diaelectricity $(0<\varepsilon<1)$ cannot exist. If one applies the thermodynamic stability criteria of the Landau-Lifshitz Eq.(21) to the ClausiusMossotti Eq.(20), then it becomes apparent that the region of stability for the model may be described by the parameter

$$
\begin{aligned}
& \eta=\left(\frac{4 \pi \alpha}{3 v}\right), \\
& \eta<1 \text { (thermodynamic stability). }
\end{aligned}
$$

Debye defines a polar molecule as one whose polarizability has a temperature dependence (in the dilute gas phase) of the form

$$
\alpha=\alpha_{0}+\left(\frac{\mu^{2}}{3 k_{B} T}\right) .
$$

By plotting $\alpha$ as a function of inverse temperature one finds a linear slope yielding the magnitude of a "permanent" dipole moment

$$
\mu=|\mathbf{p}| .
$$


If the polarizability of a single molecule (say in the gas phase) exhibits an appreciable permanent dipole moment $\mu$, then the molecule is called polar. The water molecule $\mathrm{H}_{2} \mathrm{O}$ is an important example of a polar molecule. In the dilute gas phase, a water molecule exhibits a temperature dependent polarizability of the form in Eq. (23) with

$$
\begin{aligned}
\alpha_{0}\left(\mathrm{H}_{2} \mathrm{O}\right) & \approx 1.494 \times 10^{-24} \mathrm{~cm}^{3}, \\
\mu\left(\mathrm{H}_{2} \mathrm{O}\right) & \approx 1.855 \times 10^{-18} \text { Gauss } \mathrm{cm}^{3} .
\end{aligned}
$$

For room temperature and atmospheric pressure, well known properties of water 13] include

$$
\begin{aligned}
\alpha_{\text {water }} & \approx 28.696 \AA^{3} \quad \text { (Gas Phase) } \\
v_{\text {water }} & \approx 30.014 \AA^{3} \quad \text { (Liquid Phase) }
\end{aligned}
$$

and

$$
\left.\eta_{\text {water }}=\left(\frac{4 \pi \alpha_{\text {water }}}{3 v_{\text {water }}}\right) \approx 4.0054>1 \text { (unstable }\right) .
$$

In TABLE \ we have listed some liquids along with a computation of whether or not the conventional ClausiusMossotti model yields a stable polarization disordered state.

Room temperature (and pressure) water is an important example of a substance which is in an unstable regime of the Clausius-Mossotti model. Many other polar liquids also tend to be in unstable regimes. In their studies of polar liquids, the well known physical chemists Onsager 14] and (later) Kirkwood 15, 16] tried to improve on the Clausius-Mossotti theory in such a manner that the instability would be avoided. The attempts were not entirely successful.

More recently, there have been studies which assert that polar liquids (such as water) may be found in a "superradiant" state wherein ordered domains exist. We regard these more recent proposals as likely to be true. The superradiant ordered sub-domains of the liquid carry a net polarization $\mathbf{P}_{0}$. From the viewpoint of ClausiusMossotti local fields, the polarized sub-domains should in reality be present. In deriving this result below, we shall

TABLE I: Listed below are some liquids 13]. The stability properties are evaluated on the basis of the Clausius-Mossotti model Eq. (22) with $\eta=(4 \pi \alpha / 3 v)$.

\begin{tabular}{lcl}
\hline \hline Chemical & $\eta$ & Clausius-Mossotti \\
\hline $\mathrm{C}_{2} \mathrm{H}_{5} \mathrm{OC}_{2} \mathrm{H}_{5}$ & 0.5486 & stable \\
$\mathrm{HCONH} \mathrm{H}_{2}$ & 6.1498 & unstable \\
$\mathrm{HCN}$ & 4.7479 & unstable \\
$\mathrm{HF}$ & 3.6503 & unstable \\
$\mathrm{HCONHCH}$ & 5.2093 & unstable \\
$\mathrm{C}_{6} \mathrm{H}_{5} \mathrm{CH}_{3}$ & 0.3156 & stable \\
$\mathrm{PCl}_{3}$ & 0.6728 & stable \\
$\mathrm{H}_{2} \mathrm{O}$ & 4.0054 & unstable \\
$\mathrm{CHCl}_{3}$ & 0.5538 & stable \\
\hline \hline
\end{tabular}

neglect $\alpha_{0}$ in Eq. (23) compared with the thermal term, i.e. we employ the Langevin model form

$$
\alpha_{T}=\left(\frac{\mu^{2}}{3 k_{B} T}\right) \quad(\text { Langevin })
$$

This is certainly a good approximation for water wherein $\alpha_{0}\left(H_{2} \mathrm{O}\right)<<\alpha_{\text {water }} \approx \alpha_{T}$. In what follows we shall also review how Eq.(28) is derived.

\section{ORDERED THERMODYNAMIC PHASES}

For a permanent dipole moment $\mathbf{p}=\mu \mathbf{n}$ (where $\mathbf{n}$ is a unit vector), the partition function describing the interaction with the local field $\mathbf{F}$ is given by

$$
Z=\int e^{\mathbf{p} \cdot \mathbf{F} / k_{B} T}\left(\frac{d^{2} \mathbf{n}}{4 \pi}\right)
$$

In Eq. 29), $d^{2} \mathbf{n}$ is a solid angle about the $\mathbf{n}$ direction. Explicitly,

$$
Z=\frac{1}{2} \int_{0}^{\pi} e^{\mu F \cos \theta / k_{B} T} \sin \theta d \theta
$$

yielding a free energy per unit volume of

$$
A=-\frac{k_{B} T}{v} \ln Z=-\frac{k_{B} T}{v} \ln \left[\frac{\sinh \left(\mu F / k_{B} T\right)}{\left(\mu F / k_{B} T\right)}\right] .
$$

The resulting polarization $\mathbf{P}=-(\partial A / \partial \mathbf{F})_{T}$ is parallel to $\mathbf{F}$ and has the magnitude originally derived by Langevin, i.e.

$$
P=\frac{\mu}{v}\left[\operatorname{coth}\left(\frac{\mu F}{k_{B} T}\right)-\left(\frac{k_{B} T}{\mu F}\right)\right]
$$

Note that

$$
\frac{\alpha_{T}}{v}=\lim _{F \rightarrow 0}\left(\frac{\partial P}{\partial F}\right)_{T}=\left(\frac{\mu^{2}}{3 v k_{B} T}\right),
$$

in agreement with Eq.(28).

From Eqs.(11) and (32) we may compute (in parametric form) the polarization equation of state as follows:

(i) Define the parameters

$$
\begin{aligned}
& \eta=\frac{4 \pi \alpha_{T}}{3 v}, \quad x=\frac{\mu E}{k_{B} T}, \\
& y=\frac{\mu P}{\eta k_{B} T}, \quad z=\frac{\mu F}{k_{B} T} .
\end{aligned}
$$

(ii) Eqs.(1) and (32) now read

$$
\begin{aligned}
& x=z-3 \eta\left[\operatorname{coth}(z)-\frac{1}{z}\right], \\
& y=\frac{9}{4 \pi}\left[\operatorname{coth}(z)-\frac{1}{z}\right] .
\end{aligned}
$$


(iii) By eliminating the parameter $z$ in Eqs. (35) one defines the function

$$
y=\mathcal{F}(x, \eta)
$$

and thereby the non-linear polarization equation of state

$$
P=\frac{4 \pi \mu}{9 v} \mathcal{F}\left(\frac{\mu E}{k_{B} T}, \frac{4 \pi \mu^{2}}{9 v k_{B} T}\right) .
$$

The polarization is plotted in FIG. 1 for room temperature toluene (which is disordered) and water (which is ordered).

The polarization equation of state in the LangevinClausius-Mossotti model is of the typical "mean field" variety. There exists a critical temperature

$$
T_{c}=\left(\frac{4 \pi \mu^{2}}{9 k_{B} v}\right) .
$$

Above the critical temperature, the susceptibility is positive,

$$
\left(\frac{\varepsilon_{T}-1}{4 \pi}\right)=\chi_{T}=\left(\frac{\partial P}{\partial E}\right)_{T}>0 \text { for } T>T_{c}
$$

in accordance with the Landau-Lifshitz Eq.(21). Below the critical temperature, there is an unphysical " $\chi_{T}<0$ " portion of an equation of state loop which must be subject to an "equal area" construction 17 to deduce the proper stable phase. There exists below the critical temperature a remnant polarization

$$
P_{0}(T)=\lim _{E \rightarrow 0^{+}} P(E, T)>0 \text { for } T<T_{c}
$$

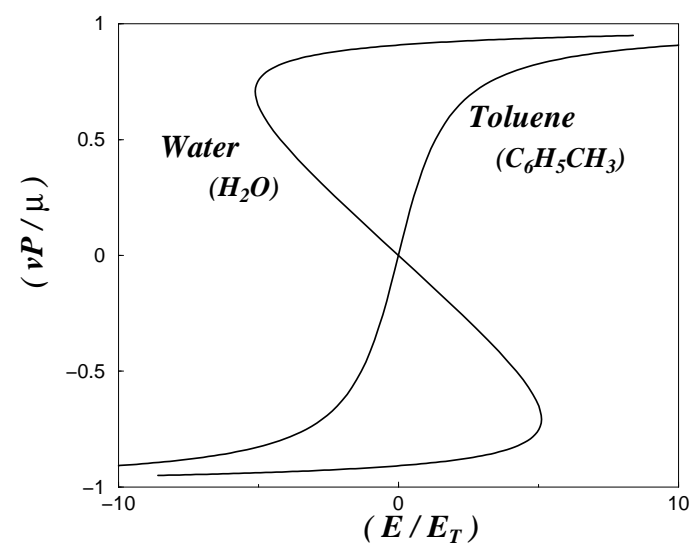

FIG. 1: The Clausius-Mossotti "dimensionless" polarization $(v P / \mu)$ is plotted as a function of the electric field, in units of the thermal electric field $E_{T}=\left(k_{B} T / \mu\right)$. Toluene is stable with a positive susceptibility $\chi_{T}=(\partial P / \partial E)_{T}$. Water is unstable as indicated by the unphysical " $\chi_{T}<0$ " portion of the loop in the equation of state. The stable phase of water may be constructed using an "equal area" rule.

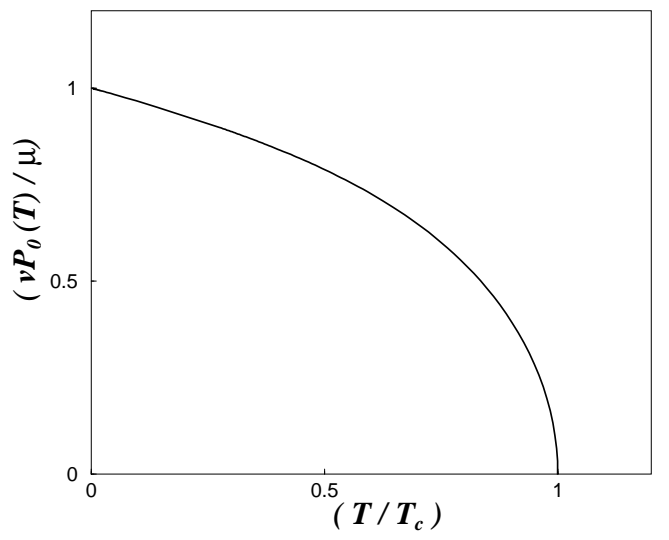

FIG. 2: Shown is the remnant polarization $P_{0}(T)$ for temperatures below the critical temperature. The ordered phase of the Langevin-Clausius-Mossotti polar liquid is ferroelectric. The ordered net polarized regions should appear in mesoscopic domains. The direction of the remnant polarization $\mathbf{n}=\left(\mathbf{P}_{0} / P_{0}\right)$ should vary depending upon the domain.

The parametric equations for the remnant polarization are given by

$$
\begin{aligned}
& \frac{T}{T_{c}}=\frac{3}{z}\left(\operatorname{coth}(z)-\frac{1}{z}\right), \\
& \frac{v P_{0}}{\mu}=\left(\operatorname{coth}(z)-\frac{1}{z}\right),
\end{aligned}
$$

where the parameter range is $0<z<\infty$. The ordered phase of the Langevin-Clausius-Mossotti polar liquid is ferroelectric with a polarization pointing in a unit vector direction $\mathbf{n}=\left(\mathbf{P}_{0} / P_{0}\right)$. The magnitude of the remnant polarization is plotted in FIG. 2.

In this picture, water at room temperature contains ordered ferroelectric domains with a polarization pointing in the direction $\mathbf{n}$. From one domain to another, the direction $\mathbf{n}$ of the polarization is changing. The situation is shown schematically in FIG. 3. Inside of a domain, there is a residual local electric field $\mathbf{F}_{0}$ and polarization $\mathbf{P}_{0}$ related by

$$
\mathbf{F}_{0}=\left(\frac{4 \pi}{3}\right) \mathbf{P}_{0}
$$

The domain picture has many interesting features only one of which we shall discuss here. If one asks what will happen to a noble gas impurity (say Argon) when it is dissolved in water, then one concludes that the atom will be drawn inside the ordered domain. The effective potential 18] on the atom is (roughly) a step function attracting the impurity into the ordered domain,

$$
\Delta U_{\text {impurity }}(\mathbf{r})=-\frac{1}{2} \alpha_{\text {impurity }}\left|\mathbf{F}_{0}(\mathbf{r})\right|^{2} .
$$

In FIG. 3 we show two of the domains with an impurity. The notion that an ordered domain is clustered 


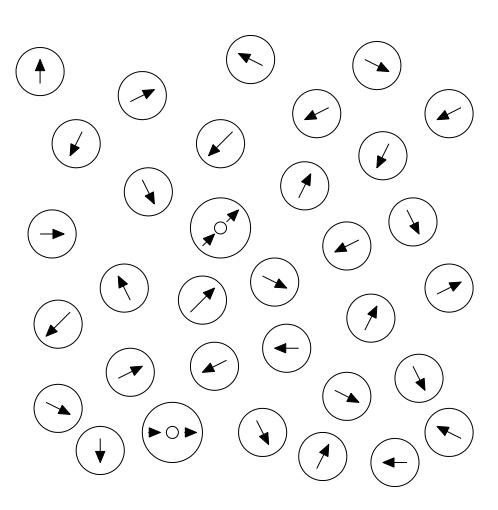

FIG. 3: Shown schematically is a system of polarized domains in water. In each domain there exists a local electric field and a polarization vector related by $\mathbf{F}_{0}=(4 \pi / 3) \mathbf{P}_{0}$. Between the ordered phase domains are unpolarized disordered water. Two of the domains are shown to contain a noble gas atom (such as Argon). The low entropy ordered domain about a noble gas impurity has been called an "iceberg" and was unexpected when it was first measured thermodynamically.

around a dissolved noble gas atom (say argon) has consequences for the thermodynamic impurity equations of state. The impurity entropy has in fact been particularly well analyzed by Frank and Evans [19, 20]. Suppose that a dilute mixture of Argon in water is such that the vapor phase mixture is in thermal equilibrium with the dilute liquid mixture. An impurity atom in water vapor is in a disordered environment. The impurity atom in liquid water will be found in an ordered environment within a domain. Impurity entropy is then lost when the impurity atom moves from the vapor to the liquid. To use the words of Frank and Evans (describing this unexpected entropy loss) "the water builds a microscopic iceberg around the non-polar molecule". There is no evidence that an ordered domain about a noble gas atom impurity is a piece of ice. The low entropy polarized domain predicted by the Langevin-Clausius-Mossotti model appears to explain the so called "iceberg effect".

\section{CONCLUSIONS}

The Clausius-Mossotti model together with the elementary statistical thermodynamics of Debye and Langevin yields a ferroelectric phase transition for many polar liquids including water. The notion of ferroelectric domains within the a polar liquid with a net polarization $\mathbf{P}_{0}$ and internal electric field $\mathbf{F}_{0}=(4 \pi / 3) \mathbf{P}_{0}$ is clearly implicit in the model and can be derived above in a fairly simple and straight forward manner. The phase transition may be described using basic concepts from elementary physics.

Nevertheless, reasoning with classical electrodynamics and classical statistical thermodynamics yields only partial insights concerning the complete microscopic theory. A detailed theory involves quantum statistical mechanics and quantum electrodynamics. For example, why does a polar $\mathrm{H}_{2} \mathrm{O}$ molecule have a permanent electric dipole moment? If one starts by calculating the quantum ground state $|0\rangle$ of the molecule employing only the non-relativistic Coulomb Hamiltonian, then parity conservation requires the vanishing of the mean dipole moment $\overline{\mathbf{p}}=\langle 0|\mathbf{p}| 0\rangle=0$. The notion of a permanent electric dipole moment requires a break in parity (space inversion) symmetry. Such a symmetry break can come about due to interactions with the quantum electrodynamic field. Similarly, the dipole-dipole interaction takes place (in the quantum electrodynamic theory) due to the exchange of virtual photons between two dipoles.

That the polarized domains have a net internal electric field $\mathbf{F}_{0}$, really means that the exchanged photons form a Bose condensate. The Bose condensate is also present in a model conventionally used to describe superradiance 21]. In spite of the long time popularity of the Clausius-Mossotti model, it was only through a study of superradiance that the ordered domains were first 22 theoretically discovered. It is hoped that the above simple Clausius-Mossotti model considerations serve to clarify the mechanism for ordering in some polar liquids.
[1] O.F. Mossotti, Memorie Mat. Fis. Modena 24, 49 (1850).

[2] R. Clausius, Die mechanische W. armtheorie II, 62 Braunschweig (1897).

[3] D.J. Griffiths, "Introduction to Electrodynamics", pp. 192 (Prentice Hall, New Jersey 1989).

[4] R. Becker, "Electromagentic Fields and Interactions", pp. 95 (Dover, New York 1982).

[5] L. Eyges, "The Classical Electromagnetic Field", pp. 111 (Dover, New York 1972).

[6] G. Preparata, QED Coherence in Matter chap. 10, pp. 195 (World Scientific, Singapore, 1995).

[7] E. Del Giudice and G. Preparata, A New QED Picture of Water in Macroscopic Quantum Coherence eds. E. Sassaroli, Y. Srivastava, J. Swain and A. Widom (World Scientific, Singapore, 1998).
[8] E. Del Guidice, G. Preparata and M. Fleischmann, J. Elec. Chem. 482, 110 (2000).

[9] S. Sivasubramanian, A. Widom and Y.N. Srivastava, Mod. Phys. Lett. B16, 1201 (2002).

[10] P. Debye, Phys. Z. 13, 97 (1912); "Polar Molecules", pp. 30 (Dover Publications Inc. New York. 1928).

[11] P. Langevin, J. Phys. 4, 678 (1905); Ann. chim. phys. 5, 70 (1905).

[12] L.D. Landau, E.M. Lifshitz, "Electrodynamics of Continuous Media", chap. II pp. 55 (Pergamon Press, New York 1975).

[13] "CRC Handbook of Chemistry and Physics", (CRC Press, Cleveland, OH, 2001).

[14] L. Onsager, J. Amer. Chem. Soc. 58, 1486 (1936).

[15] J.G. Kirkwood, J. chem. Phys. 7, 911 (1939); G. Oster 
and J.G. Kirkwood, J. chem. Phys. 11, 175 (1943); J.G. Kirkwood, Trans. Faraday Soc. A42, 7 (1946).

[16] H. Fröhlich, "Theory of Dielectrics", Oxford University Press (1949).

[17] B.A. Strukov and A.P. Levanyuk, "Ferroelectric Phenomena in Crystals", pp. 57 (Springer, New York 1997).

[18] L.D. Landau, E.M. Lifshitz, "Quantum Mechanics", chap. XI pp. 341 (Pergamon Press, New York 1981).

[19] H.S. Frank and M.W. Evans, J. Chem. Phys. 13, 507
(1945)

[20] R.A. Robinson and R.H. Stokes, "Electrolyte Solution", Second Edition chap. I pp. 14 (Dover, New York 2002).

[21] K. Hepp and E.H. Lieb, Phys. Rev. A8, 2517 (1973); Ann. Phys. 76, 360 (1973).

[22] E. Del Giudice, G. Preparata and G. Vitiello, Phys. Rev. Lett. 61, 1085 (1988) 